\title{
THE GAUSS MAP ON A CLASS OF INTERVAL TRANSLATION MAPPINGS
}

\author{
H. BRUIN AND S. TROUBETZKOY
}

\begin{abstract}
We study the dynamics of a class of interval translation map on three intervals. We show that in this class the typical ITM is of finite type (reduce to an interval exchange transformation) and that the complement contains a Cantor set. We relate our maps to substitution subshifts. Results on Hausdorff dimension of the attractor and on unique ergodicity are obtained.
\end{abstract}

\section{INTRODUCTION}

In the last years there has been an increased interest in the study of piecewise isometries, see e.g. [AKT, ACP, G, \&, LV, M, V1, V2] and the references therein. Classically, invertible piecewise isometries have been studied in one dimension; these are the so-called interval exchange mappings (IETs for short), which can appear for example as first return maps of geodesic and billiard flows. A natural generalization of IETs to the noninvertible case, interval translation mappings (ITMs for short), has been recently introduced by Boshernitzan and Kornfeld [BK]. ITMs have been studied for their topological dynamics in [ST], and for their invariant measures and complexity in [BH]. Many 0-entropy maps of the interval are (semi)conjugate to ITMs BH]. In [BK] several very interesting questions were asked, which we answer in this article for a special class of interval translation mappings.

We define a class of ITMs which can be viewed as translations of two intervals on the circle. The example given in [BK is a special member of our class. This example was the first one for which a Cantor attractor for an ITMs was observed. We define an inducing procedure similar to Rauzy induction for interval exchange mappings [R, V1]. It defines a map $G$ in parameter space which plays the same role as the well-known Gauss map for circle rotations; we call $G$ the Gauss map for our class of ITMs. Whether the induction procedure can be extended to all ITMs is a very interesting open problem.

Using the Gauss map, we prove that almost every map in our class is of finite type (i.e., its attractor is a union interval rather than a Cantor set), giving a partial answer to a question posed in BK. If the ITM has a Cantor

2000 Mathematics Subject Classification. Primary: 37E05, 37B10 Secondary: 37A05, $37 \mathrm{~B} 40$

Key words and phrases. interval translation map, interval exchange transformation, substitution shift, unique ergodicity, Hausdorff dimension.

We gratefully acknowledge the support of Université de Toulon et du Var, the Royal Netherlands Academy of Arts and Sciences (KNAW) and Van Gogh NWO/EGIDE . 
attractor, we specify an isomorphism to a shift space generated by a chain of substitutions. We give an upper bound on the dimension of the attractor. Although the attractor is "dynamically defined", it is interesting to note that its upper box dimension need not be equal to its Hausdorff dimension. Hausdorff measure, whether finite or not, is always $T$-invariant. Finally we give sufficient conditions for mappings in our class to be uniquely ergodic, and a sufficient condition preventing unique ergodicity.

In a companion paper, J. Cassaigne shows that the (subword) complexity $p(n)$ of the subshift describing our system is linear, in fact $p(n) \leq 3 n$ [C2]. This yields a partial answer to another question posed in BK. Cassaigne's original technique [C1] is designed for substitution subshifts. In [C2], he extends the method for the chain of substitutions developed in our paper.

\section{Statement of Results}

Let $0=\beta_{0}<\beta_{1}<\cdots<\beta_{r}=1, I=[0,1)$ and, for $i=0, \ldots, r, B_{i} \stackrel{\text { def }}{=}$ $\left[\beta_{i-1}, \beta_{i}\right)$. An interval translation mapping is an interval map $T: I \rightarrow I$ given by

$$
T(x) \stackrel{\text { def }}{=} x+\gamma_{i} \text { if } x \in B_{i},
$$

where $\gamma_{i}$ are fixed numbers such that $T$ maps $I$ into itself. We also define the image of 1 by $T(1) \stackrel{\text { def }}{=} \lim _{x \rightarrow 1^{-}} T(x)$.

Define $\Omega_{0}=I$ and $\Omega_{n}=T\left(\Omega_{n-1}\right)$. The set $\Omega \stackrel{\text { def }}{=} \cap_{n} \Omega_{n}$ is called the attractor of the ITM. We say that $T$ is of finite type if $\Omega_{n}=\Omega_{n+1}$ for some $n$. In this case, the attractor $\Omega=\Omega_{n}$ is a finite union of intervals, and $\left.T\right|_{\Omega}$ is an interval exchange transformation (IET). If $\Omega_{n+1}$ is strictly smaller than $\Omega_{n}$ for each $n$, then $\bar{\Omega}$ is a Cantor set or the union of a Cantor set and a finite collection of intervals. The latter only happens when $T$ is reducible; orbits do not visit both the Cantor set and the intervals. We will ignore this reducible case, and concentrate on the case that $\bar{\Omega}$ is a Cantor set; $T$ is said to be of type $\infty$ in this case. For convenience, we will often consider $\bar{\Omega}$ instead of $\Omega$ to be the attractor, and assume that $\left.T\right|_{\bar{\Omega} \backslash \Omega}$ is (re)defined by continuity from the left.

Our first result is a general structure theorem about the topological dynamics of ITMs. In fact, this theorem is a consequence of results from [HR], but since that proof requires extensive machinery and is spread out over several papers, we prefer to give a direct proof for our case, which is more in the spirit of the well-known results for IETs, see [KH].

Theorem 1. If $\left.T\right|_{\bar{\Omega}}$ is transitive, then this restriction is minimal.

It follows from ST that $\bar{\Omega}$ is a Cantor set when $T$ is of infinite type. We have

Theorem 2. Let $d$ be the Hausdorff dimension of $\bar{\Omega}$. The Hausdorff $d$ dimensional measure $H_{d}$ on $\bar{\Omega}$ is T-invariant. 
Remark: this theorem is a new result for ITMs of infinite type. In general we do not know if $0<H_{d}(\bar{\Omega})<\infty$. In fact, if $d=0$ then $H_{d}(\bar{\Omega})$ is infinite. Examples of ITMs for which $d=0$ are produced in Theorem 10 .

The main results of this article are on interval translation maps of a special form. Consider $U \stackrel{\text { def }}{=}\{(\alpha, \beta): 0 \leq \beta \leq \alpha \leq 1\}, L \stackrel{\text { def }}{=}\{(\alpha, \beta): 0 \leq \alpha \leq$ $\beta+1 \leq 1\}$ and $R \stackrel{\text { def }}{=} U \cup L$. For $(\alpha, \beta)$ in the interior $U^{\circ}$ of $U$ consider the ITM $T=T_{\alpha, \beta}:[0,1) \rightarrow[0,1)$ defined by (see Figure 1 )

$$
T(x) \stackrel{\text { def }}{=} \begin{cases}x+\alpha & \text { for } x \in[0,1-\alpha) \\ x+\beta & \text { for } x \in[1-\alpha, 1-\beta) \\ x+\beta-1 & \text { for } x \in[1-\beta, 1) .\end{cases}
$$

By identifying the points 0 and 1 we get an interval translation map of

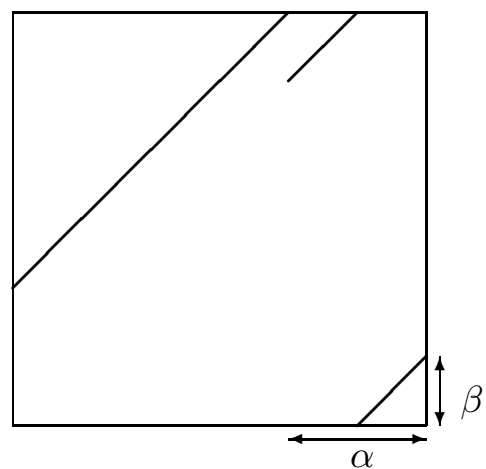

Figure 1. The map $T_{\alpha, \beta}$.

the circle with two intervals. In the three-parameter class of all ITMs on the circle with two intervals, the condition that $\lim _{y \rightarrow \beta_{1}^{-}} T(y)=\beta_{3}$ lets us consider this two parameter subfamily. A special example of an ITM of this form was considered in BK.

Proposition 3. If $T_{\alpha, \beta}$ is aperiodic then its restriction to $\bar{\Omega}$ is minimal. If a single orbit of $T_{\alpha, \beta}$ is finite, then every orbit is eventually periodic and the restriction to the attractor is isomorphic to a rational circle rotation.

Proposition 4. The map $T_{\alpha, \beta}$ is of finite type if and only if there is an interval $J$ such that $T_{\alpha, \beta}$ induced on $J$ is an interval exchange of two intervals, i.e., $T_{\alpha, \beta}$ is isomorphic to a circle rotation. If $T_{\alpha, \beta}$ is aperiodic and of finite type, then it is uniquely ergodic.

We remark that if $\alpha=1$ or $\alpha=\beta$ then $T$ is a circle rotation, while if $\beta=0$ then $T$ is a noninvertible ITM on two intervals and in this case the identity on its attractor.

Proof of Proposition 4. We begin by proving the if statement. By Proposition 3, if $T$ has a periodic point then it is of finite type. Thus we assume that $T$ is aperiodic and therefore minimal on $\bar{\Omega}$ by Proposition 3 . Let 
$J$ be an interval such that the induced map $T_{J}$ on $J$ is an interval exchange of two intervals. We have $J \subset \Omega$ and thus $T$ is of finite type since for any transitive ITM of infinite type $\Omega$ must be a Cantor set [ST].

We prove the only if statement by contradiction. Consider the first return map $\hat{T}=T_{\Delta_{1}}$ of $T_{\alpha, \beta}$ to the interval $\Delta_{1}:=[1-\alpha, 1)$. Clearly we have $\left.\hat{T}\right|_{[1-\alpha, 1-\beta)}=\left.T\right|_{[1-\alpha, 1-\beta)}$. There are two cases for the interval $[1-\beta, 1)$.

i) The whole interval $[1-\beta, 1)$ returns at the same time. In this case the map $\hat{T}$ is an interval translation map on two intervals. Thus $\hat{T}$ is an interval exchange of two intervals.

ii) There are two different return times, that is, there is a positive integer $k$ such that the left part of the interval returns in $k$ steps and the right part of the interval returns in $k-1$ steps. In this case the map $\hat{T}$ of the form $T_{\alpha^{\prime}, \beta^{\prime}}$ where

$$
\left(\alpha^{\prime}, \beta^{\prime}\right) \stackrel{\text { def }}{=} G(\alpha, \beta) \stackrel{\text { def }}{=}\left(\frac{\beta}{\alpha}, \frac{\beta-1}{\alpha}+\left\lfloor\frac{1}{\alpha}\right\rfloor\right)
$$

where $k=\left\lfloor\frac{1}{\alpha}\right\rfloor$.

Repeating the inducing procedure we either are always in case ii) or at some time step we reach case i), and then there is a subinterval $J$ such that the first return map on $J$ is an interval exchange of two intervals. If we are always in case ii) then the induced map to $\Delta_{n}:=\left[1-\prod_{i=0}^{n-1} \alpha_{i}, 1\right)$ (where $\left.\left(\alpha_{i}, \beta_{i}\right) \stackrel{\text { def }}{=} G^{i}(\alpha, \beta)\right)$ is not invertible. This means that for each $n$ the set $\Delta_{n}$ is not contained in $\Omega$. Since $\Omega \cap \Delta_{n}=\cup_{i \geq 0} T_{\Delta_{n}}^{i}\left(\Omega \cap \Delta_{n+1}\right)$ this implies that $\Omega$ cannot contain any interval and thus $T$ is of infinite type.

Aperiodic circle rotations are always uniquely ergodic. Since the Kakutani tower of $T_{\alpha, \beta}$ over the induced map is finite, the map $T_{\alpha, \beta}$ is uniquely ergodic if and only if the induced rotation is uniquely ergodic.

Equation (1) defines the Gauss map $G: U^{\circ} \rightarrow R$. To understand the action of this map consider the region $\mathcal{U}_{k} \stackrel{\text { def }}{=}\left\{(\alpha, \beta) \in U: \frac{1}{k+1}<\alpha \leq \frac{1}{k}\right\}$, see Figure 2. For each $k \in \mathbb{N}, G$ maps $\mathcal{U}_{k}$ into $R$ in the following way: the right boundary of $\mathcal{U}_{k}$ is mapped onto the top boundary of $R$, the left boundary of $\mathcal{U}_{k}$ is mapped onto the bottom boundary of $R$, the bottom boundary of $\mathcal{U}_{k}$ is mapped onto the left boundary of $R$ and the top boundary of $\mathcal{U}_{k}$ is mapped onto the right boundary of $R$. Note that if $(\alpha, \beta) \in \partial \mathcal{U}_{k}$ for some $k$, then $T_{G(\alpha, \beta)} \in \partial(R)$, thus of finite type and consequently $T_{\alpha, \beta}$ is also of finite type. Let

$$
A \stackrel{\text { def }}{=} \cap_{n \geq 0} G^{-n}\left(U^{\circ}\right)
$$

see Figure 3 .

Corollary 5. $T_{\alpha, \beta}$ is of infinite type if and only if $(\alpha, \beta) \in A$.

Proof. If $(\alpha, \beta) \notin A$ then at some step of the inducing procedure $G^{n}(\alpha, \beta) \in$ $L \cup \partial U$ and the induced map is an ITM on two intervals. 

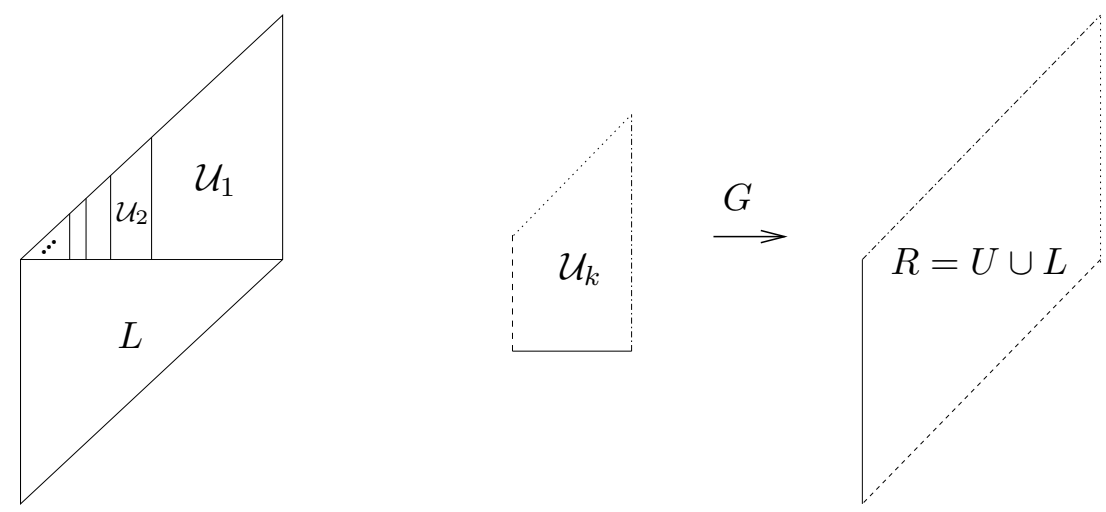

Figure 2. The action of the map $G$.

If $(\alpha, \beta) \in A$ then the inducing procedure can be repeated indefinitely and thus $T$ is of infinite type by the proof of Proposition 4 .

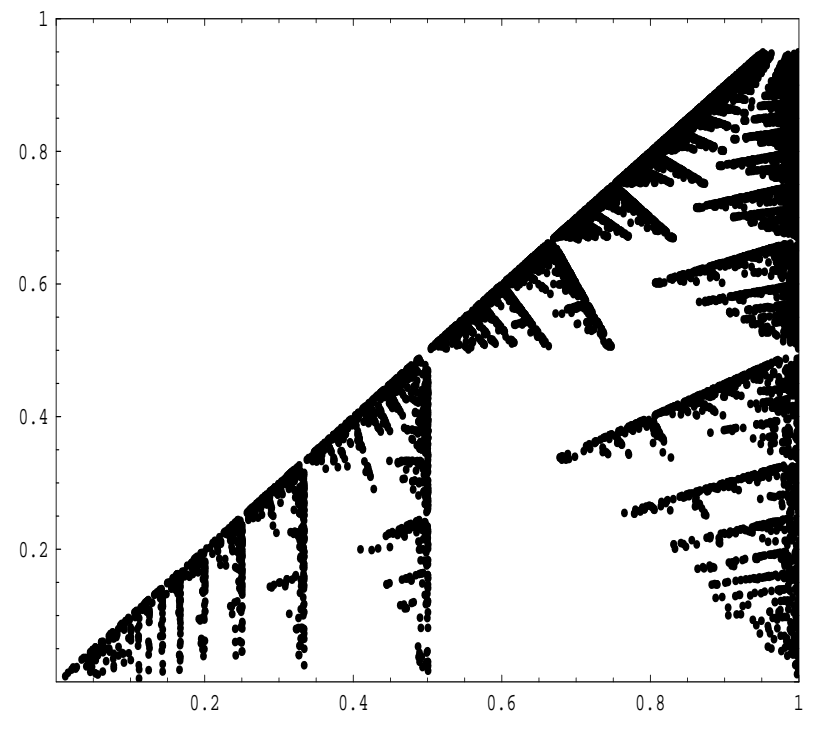

Figure 3. Approximation of the set $A$ based on an iterated function system with 10,000 pixels. The black should reach up to the top right corner $(1,1)$, but does not because this is a neutral fixed point of $G$ (see Proposition 15).

Theorem 6. The set $A$ has Lebesgue measure 0. In particular, for Lebesgue almost every $(\alpha, \beta) \in U$ the map $T_{\alpha, \beta}$ is of finite type and aperiodic.

Let $\mathbb{N}=\{1,2,3, \ldots\}$. The starting result of the analysis of our family of ITMs is: 
Theorem 7. The set $A$ is uncountable and is naturally indexed by $\mathcal{K} \stackrel{\text { def }}{=}\left\{k=k_{0} k_{1} \ldots ; k_{i} \in \mathbb{N}, k_{2 i} \neq 1\right.$ inf. often and $k_{2 i+1} \neq 1$ inf. often $\}$.

for any sequence $k$, the map $\left.T_{\alpha, \beta}\right|_{\Omega}$ is isomorphic to the shift space $\left(\Sigma_{\alpha, \beta}, \sigma\right)$ generated by the chain of substitutions $\chi_{k_{0}} \circ \chi_{k_{1}} \circ \chi_{k_{2}} \circ \cdots$ where

$$
\chi_{k} \stackrel{\text { def }}{=}\left\{\begin{array}{l}
1 \rightarrow 2 \\
2 \rightarrow 31^{k} \\
3 \rightarrow 31^{k-1}
\end{array}\right.
$$

We will call an ITM self-similar if there exists one and hence infinitely many subintervals $\Delta \neq I$ such that the induced mapping $T_{\Delta}: \Delta \rightarrow \Delta$ differs from $T$ only by affine scaling.

Proposition 8. The set of periodic points of $G$ is countable and dense in $A$. The set $\Omega$ is self-similar for any $(\alpha, \beta)$ which is $G$-periodic.

For each $x \in \Omega$ define the itinerary $e(x) \stackrel{\text { def }}{=} e_{0}(x) e_{1}(x) \ldots$ where $e_{k}(x)=j$ iff $T^{k}(x) \in B_{j}=\left[\beta_{j-1}, \beta_{j}\right)$. Let $\Sigma=\overline{e(\Omega)}$. The subshift $\Sigma$ is called adic if it is generated by a sequence $l$, where $l=\lim _{n} \sigma_{1} \circ \sigma_{2} \circ \cdots \circ \sigma_{n}(a)$, and the substitutions $\sigma_{i}$ are primitive and taken from a finite collection.

Proposition 9. Let $(\alpha, \beta) \in A$. If the $G$-orbit of $(\alpha, \beta)$ does not accumulate at the points $(0,0)$ or $(1,1)$ then $T_{\alpha, \beta}$ is adic. This set of $(\alpha, \beta)$ is uncountable and dense in $A$.

Write $\operatorname{dim}_{H}, \overline{\operatorname{dim}_{B}}$ and $\underline{\operatorname{dim}_{B}}$ for Hausdorff dimension and upper resp. lower box dimension.

Theorem 10. There exists $r=0.84955 \cdots<1$ such that for all $(\alpha, \beta) \in A$,

$$
\overline{\operatorname{dim}_{\mathrm{B}}}\left(\bar{\Omega}_{\alpha, \beta}\right) \leq r .
$$

There exist maps $T_{\alpha, \beta}$ such that $\operatorname{dim}_{\mathrm{H}}\left(\bar{\Omega}_{\alpha, \beta}\right)=\underline{\operatorname{dim}_{\mathrm{B}}}\left(\bar{\Omega}_{\alpha, \beta}\right)=0$, as well as maps where $0=\operatorname{dim}_{\mathrm{H}}\left(\bar{\Omega}_{\alpha, \beta}\right)<\overline{\operatorname{dim}_{\mathrm{B}}}\left(\bar{\Omega}_{\alpha, \beta}\right)$.

Results of Boshernitzan imply that $\operatorname{dim}_{\mathrm{H}}(\mu) \geq \frac{1}{2}$ for any $T$-invariant Borel probability measure for almost every $\alpha, \beta$ and for any $\alpha, \beta$ which are algebraic [Bo1]. The Hausdorff dimension of $\bar{\Omega}$ has been computed before in BK for one particular example. Let

$$
P_{k}(x)=x^{3}-x^{2}-k x+1 .
$$

Denote the roots of $P_{k}$ by $\underline{r}_{k}<r_{k} \leq \bar{r}_{k}$. One can check that $\underline{r}_{k} \approx-\sqrt{k}+\frac{1}{2}$, $r_{k} \approx \frac{1}{k}$ and $\bar{r}_{k} \approx \sqrt{k}+\frac{1}{2}$ for large $k$. If $\alpha_{k}=r_{k}$, then $\left(\alpha_{k}, \alpha_{k}^{2}\right)$ is a fixed point of $G$, and the Hausdorff dimension of $\bar{\Omega}_{\alpha_{k}, \alpha_{k}^{2}}$ equals $-\frac{\log \left(\bar{r}_{k}\right)}{\log r_{k}}$. For the case $k=3$, the details have been worked out in [BK]. If $k \rightarrow \infty$, then $\operatorname{dim}_{\mathrm{H}}\left(\bar{\Omega}_{\alpha_{k}, \alpha_{k}^{2}}\right) \rightarrow \frac{1}{2}$.

As we have seen in Proposition 4 , every $T_{\alpha, \beta}$ of finite type is isomorphic to a circle rotation, and therefore uniquely ergodic. If $T_{\alpha, \beta}$ satisfies the hypotheses of Proposition 9 then unique ergodicity is relatively easy to prove. 
Fluctuations combined with large values in the sequence $\left(k_{i}\right)$ complicate a general analysis. Both cases occur:

Theorem 11. Let $T_{\alpha, \beta}$ be of type infinity, with sequence $\left(k_{i}\right)$ as in Theorem \$. If for some $\lambda>1, k_{i} \geq \lambda k_{i-1}$ for all $i$ sufficiently large, then $T_{\alpha, \beta}$ is not uniquely ergodic.

Theorem 12. Let $T_{\alpha, \beta}$ be of type infinity, with sequence $\left(k_{i}\right)$ as in Theorem 匇. Let $L_{2 i}=\min \left\{r \geq 1 ; k_{2 i+r} \neq 1\right\}$. If

$$
\sum_{i} \frac{k_{2 i}-1}{k_{2 i}} \sqrt{\frac{1}{k_{2 i-1} L_{2 i}}}=\infty,
$$

or

$$
\prod_{i \geq 1} \frac{k_{2 i}}{k_{2 i-1}+\frac{1}{L_{2 i}}}=0,
$$

(or either condition holds with $2 i$ replaced by $2 i-1$ ), then $T_{\alpha, \beta}$ is uniquely ergodic.

Conditions (3) and (4) have a non-empty symmetric difference. For instance, if $k_{2 i}=2^{i}, k_{2 i-1}=3^{i}$, then (任) applies and not (3), while the case $k_{i}=i+1$ is covered only by Condition (3). Theorem 12 allows a corollary on the abundance of uniquely ergodic ITMs in our class.

Corollary 13. The set $A_{U E}:=\left\{(\alpha, \beta) \in A \mid T_{\alpha, \beta}\right.$ is uniquely ergodic $\}$ is a dense $G_{\delta}$ set in $A$, i.e., for each compact set $K \subset A, A_{U E} \cap K$ is dense $G_{\delta}$.

The method for proving that the map $T_{\alpha, \beta}$ is uniquely ergodic or not has the same flavor as the method that Keane [ $\mathrm{K}]$ used for certain IET's. In fact, it gives also a way to estimate the number of ergodic measures, and so we retrieve a result proven in more generality by Buzzi and Hubert [BH].

Corollary 14. Each map $T_{\alpha, \beta}$ has at most two ergodic invariant measures.

\section{TRANSITIVE IMPLIES MINIMAL}

In this section we give a direct proof of Theorem 1 .

Proof of Theorem 1. If $T$ is of finite type, i.e., if $\left.T\right|_{\Omega}$ is an IET, minimality is well known consequence of transitivity (see for example Corollary 14.5.11 in [KH]). So assume that $T$ is of type $\infty$. Let $y \in \bar{\Omega}$ be such that $\bar{\Omega}$ is $\omega(y)$, the omega limit set of $y$.

Claim 1: There exists $0<i<r$ and an interval $J \subset I$ such that $J \cap \bar{\Omega} \neq \emptyset$ and $J \cap \bar{\Omega} \subset \omega\left(\beta_{i}\right)$.

Note that $\beta_{0}$ and $\beta_{r}$ are not discontinuity points of the map $T$ and therefore not necessary in Claim 1.

Start with $i=1$ and $J_{1}=I$. Clearly $J_{1} \cap \bar{\Omega} \neq \emptyset$. If $J_{1} \cap \bar{\Omega} \subset \omega\left(\beta_{1}\right)$, then we are finished. Otherwise, there exists an interval $J_{2} \subset J_{1}$, intersecting $\bar{\Omega}$, such that $\omega\left(\beta_{1}\right) \cap J_{2}=\emptyset$. Next check if $J_{2} \cap \bar{\Omega} \subset \omega\left(\beta_{2}\right)$. If so, then 
we are finished. Otherwise, there exists an interval $J_{3} \subset J_{2}$, intersecting $\bar{\Omega}$, such that $\omega\left(\beta_{2}\right) \cap J_{3}=\emptyset$. Continue this way. Since there are only finitely many discontinuity points, we arrive at some interval $J_{s}$, intersecting $\bar{\Omega}$ but disjoint from $\omega\left(\beta_{i}\right)$ for all $0 \leq i<r$. But this implies that $J_{s} \subset \Omega_{n}$ for all $n$, and $T$ cannot be of type $\infty$.

Claim 2: There exists $0<i<r$ such that $\bar{\Omega}=\omega\left(\beta_{i}\right)$.

Let $i$ and $J$ be from Claim 1 . Since $\operatorname{orb}(y)$ is dense in $\bar{\Omega}$, there exists $T^{k}(y) \in J$. Therefore $\omega\left(\beta_{i}\right) \supset \omega\left(T^{k}(y)\right) \supset \bar{\Omega}$.

Claim 3: $\bar{\Omega}=\omega\left(\beta_{i}\right)$ for each $0<i<r$.

Let $i_{0}$ be the $i$ from Claim 2. Let $X_{i}=\left\{\beta_{j} ; 0<j<r\right.$ and $\left.\beta_{j} \in \omega\left(\beta_{i}\right)\right\}$. Clearly, $X_{i_{0}}=\left\{\beta_{1}, \ldots, \beta_{r-1}\right\} \cap \bar{\Omega}$. Note that if $\beta_{j} \in X_{i}$, then $X_{j} \subset X_{i}$, and also that $X_{i} \neq \emptyset$. Indeed, if $X_{i}=\emptyset$, then there exists a neighborhood $U$ of $T\left(\beta_{i}\right)$ such that $T^{k}(U) \not \supset \beta_{j}$ for any $0<j<r$ and any $k \geq 0$. This implies that any $\Omega_{n}$ contains an interval of length $\geq|U|$, contradicting that $T$ is of type $\infty$.

To prove the claim, we need to show $X_{i}=X_{i_{0}}$ for each $i$. Assume by contradiction that $X_{i_{1}}$ is the/a largest set strictly smaller than $X_{i_{0}}$. Find $\varepsilon>0$ such that $\left|T^{k}\left(\beta_{i}\right)-\beta_{j}\right|<\varepsilon$ implies $\beta_{j} \in X_{i}$. Find $\beta \in \operatorname{orb}\left(\beta_{i_{1}}\right)$ and $z \in \operatorname{orb}(y)$ such that $|\beta-z|<\varepsilon / 2$ and iterate these points. Whenever some $\beta_{j} \in\left(T^{k}(\beta), T^{k}(z)\right)$ (in particular, $\beta_{j} \in X_{i_{1}}$ ), continue iterating with $T^{k}(z)$ and $\beta_{j}$. We get that each point in $\operatorname{orb}(z)$ is no more than $\varepsilon / 2$ away from some iterate of some point $\beta_{j} \in X_{i_{1}}$. Since $\operatorname{orb}(z)$ approximates every point in $X_{i_{0}}$ arbitrarily closely, it follows that $X_{i_{1}}=X_{i_{0}}$.

Now we can finish the proof. Take $x \in \bar{\Omega}$ arbitrary. No neighborhood $U \ni T(x)$ can be iterated indefinitely without being cut, or otherwise $T$ is not of type $\infty$. Therefore there exists $0<j<r$ such that $\beta_{j} \in \omega(x)$. By Claim 3, $\omega(x) \supset \bar{\Omega}$.

Proof of Proposition 3. Theorem 2.4 of [ST] states that $\left.T_{\alpha, \beta}\right|_{\Omega}$ is minimal when it is aperiodic.

If there is a finite orbit, then there is an interval of periodic orbits. Let $n$ be the period. We consider $T$ as a mapping of the circle, thus the point $1-\beta$ is a point of continuity of $T$. Let $[a, b)$ a maximal interval such that $T^{i}$ is continuous on $[a, b)$ for $i=1,2, \ldots n$ and $T^{n}$ is the identity. Let $B_{0}:=\cup_{i=0}^{n-1} T^{i}[a, b)$, the set $B_{0}$ consists of $n$-periodic points. By maximality, the points $1-\alpha$ and 1 must be right end points of two of the intervals which make up $B_{0}$. Thus, it follows that $T$ has no other periodic points.

Let $B:=\cup_{i=0}^{\infty} T^{-i} B_{0}$ and $C:=I \backslash B$. The set $B$ is the basin of attraction of the periodic component $A$; it consists of a countable union of half open intervals. The set $C$ is $T$-invariant.

Suppose that the second statement in the proposition is not true, i.e., the set $C$ is non-empty. 
The proof of this proposition is now a modification of the proof of Theorem 2.4 of [ST]. This theorem gives a sharp upper bound on the number of minimal sets for an aperiodic ITM. We remind the reader of the notation. Let $D:=\left\{\beta_{0}, \ldots, \beta_{r-1}\right\}$ and for an interval $J$ let $D(J):=\left\{\beta_{i} \in D\right.$ : $T^{n} \beta_{i} \in \operatorname{int}(J)$ for some $\left.n \geq 0\right\}$. We called an interval $J$ a minimal interval if $D(J) \neq \emptyset$ and $D(J)=D\left(J_{1}\right)$ for all subintervals $J_{1}$ of $J$. In the proof of Theorem 2.4 it was shown that under the assumption of aperiodicity minimal intervals induce a partition of $D$ and that there is no minimal interval $J$ for which $D(J)$ consists of a single $\beta_{i}$.

If we drop the assumption of aperiodicity, then the same proof shows that minimal intervals induce a partition of those $\beta_{i} \in D$ whose orbit is not periodic or preperiodic. Furthermore, in our case since $1-\beta$ is a point of continuity we can assume $D:=\{0,1-\alpha\}$. However, one of these two points must be the left endpoint of one of the interval making up $B_{0}$, thus it has a periodic orbit. Thus no minimal interval can exist since for every minimal interval $D(J)$ consists of at least two points.

\section{Properties of the Gauss map (Rauzy induction)}

In this section we collect some metric properties of the map $G$ and describe the structure of the set $A$.

Proposition 15. The second iterate of the map $G$ is uniformly expanding outside any neighborhood of the neutral fixed point $(1,1)$.

Proof. The map $G$ is infinite-to-one. Away from its singularities the Jacobian of $G$ is

$$
D G=\left(\begin{array}{cc}
\frac{-\beta}{\alpha^{2}} & \frac{1}{\alpha} \\
\frac{1-\beta}{\alpha^{2}} & \frac{1}{\alpha}
\end{array}\right)
$$

with determinant $-\alpha^{-3}$. The eigenvalues of $D G$ are

$$
\lambda^{ \pm} \stackrel{\text { def }}{=} \frac{1}{\alpha} \cdot\left(\frac{\alpha-\beta}{2 \alpha} \pm \sqrt{\left(\frac{\alpha-\beta}{2 \alpha}\right)^{2}+\frac{1}{\alpha}}\right) .
$$

The point $(1,1)$ is the only nonexpanding fixed point of $G$ : it is elliptic with eigenvalues \pm 1 . The eigenvalue +1 does not occur at any other point in $R$ (it occurs on the curve $\beta=\alpha^{-1}+\alpha-\alpha^{2}$ ), while the eigenvalue -1 occurs exactly on the intersection of the curve $\beta=\alpha^{2}+\alpha-\alpha^{-1}$ with $R$. Because of the influence of this curve we consider the map $G^{2}((\alpha, \beta))=\left(\alpha^{\prime \prime}, \beta^{\prime \prime}\right)$ where

$$
\left(\alpha^{\prime \prime}, \beta^{\prime \prime}\right)=\left(\frac{\alpha}{\beta} \cdot\left(1+\left\lfloor\frac{1-\alpha}{\alpha}\right\rfloor\right)+\frac{\beta-1}{\beta}, \frac{2 \beta-1}{\beta}+\frac{\alpha}{\beta}\left\lfloor\frac{1-\alpha}{\alpha}\right\rfloor+\left\lfloor\frac{\alpha-\beta}{\beta}\right\rfloor\right) .
$$

The Jacobian of $G^{2}$ is

$$
D G^{2}=\left(\begin{array}{cc}
\frac{1}{\beta}\left(1+\left\lfloor\frac{1-\alpha}{\alpha}\right\rfloor\right) & \frac{-\alpha}{\beta^{2}}\left(1+\left\lfloor\frac{1-\alpha}{\alpha}\right\rfloor\right)+\frac{1}{\beta^{2}} \\
\frac{1}{\beta\left\lfloor\frac{1-\alpha}{\alpha}\right\rfloor} & \frac{1}{\beta^{2}}+\frac{-\alpha}{\beta^{2}}\left\lfloor\frac{1-\alpha}{\alpha}\right\rfloor
\end{array}\right)
$$


and the determinant is $\beta^{-3}$. Recall that $k=1+\left\lfloor\frac{1-\alpha}{\alpha}\right\rfloor$. The eigenvalue of $D G^{2}$ are

$$
\Lambda^{ \pm} \stackrel{\text { def }}{=} \frac{1+\alpha(1-k)+\beta k \pm \sqrt{(1+\alpha(1-k)+\beta k)^{2}-4 \beta}}{2 \beta^{2}} .
$$

For $k=1$, we get $\Lambda^{+}=1 / \beta^{2}$ and $\Lambda^{-}=1 / \beta$. Note also that $\Lambda^{-}=1 / \alpha$ on the line $\alpha=\beta$. For the general case, the equation $\Lambda^{-}>1$ is equivalent to

$$
\left[(1+\alpha(1-k)+\beta k)-2 \beta^{2}\right]^{2}>(1-\alpha(1-k)+\beta k)^{2}-4 \beta,
$$

which follows from $1+\beta^{3}>\beta(1-\alpha(1-k)+\beta k)$. Since $\beta \leq \alpha \leq 1 / k$ this is easily checked to be true for all $\beta \in(0,1)$. Hence $G^{2}$ is hyperbolic expanding outside a neighborhood of the fixed point $(1,1)$.

Proof of Theorem 6. Let $V \stackrel{\text { def }}{=} U^{\circ} \cap G^{-1} U^{\circ}$. Clearly $A=\cap_{n \geq 0} G^{-2 n}(V)$. Fix a small neighborhood $N_{\varepsilon}$ of the point $(1,1)$. Consider the first return map $F_{\varepsilon}$ to the set $U \backslash N_{\varepsilon}$. Consider $A_{\varepsilon} \stackrel{\text { def }}{=} \cap_{n \geq 0} F_{\varepsilon}^{-2 n}\left(U_{1} \backslash N_{\varepsilon}\right)$. We have $A=(1,1) \cup \bigcup_{\varepsilon>0} A_{\varepsilon}$ since the elliptic point $(1,1)$ is weakly repelling for $G$. Since $F_{\varepsilon}^{2}$ is uniformly expanding the set $A_{\varepsilon}$ has zero measure, and thus $A$ has zero measure as well.

It immediately follows that $T_{\alpha, \beta}$ is of finite type for Lebesgue a.e. $(\alpha, \beta)$. However, among the finite type parameters, the (eventually) periodic ones form a countable union of smooth curves, so $T_{\alpha, \beta}$ is aperiodic for a.e. $(\alpha, \beta)$.

Proof of Theorem 7. We can give symbolic dynamics for the set $A$. The sets $\mathcal{U}_{k}$ from Figure 2 form a Markov partition for the map $\left.G\right|_{A}$. If $(\alpha, \beta) \in \partial \mathcal{U}_{k}$ for some $k$, then $T_{\alpha, \beta}$ is of finite type. Indeed, if $(\alpha, \beta) \in \partial U_{k}$ is on the bottom or left boundary (excepting the points $(0,0)$ and $(1,0)$ ) then $G(\alpha, \beta) \notin U$ so the code of $(\alpha, \beta)$ is not defined. On the other hand if $(\alpha, \beta) \in \partial U$ is on the top or right boundary then $G(\alpha, \beta)$ belongs to either the upper boundary or the right boundary of the triangle $U$ and $G$ permutes these two sets. Hence the code of $(\alpha, \beta)$ will eventually have a 1 at every other position. It follows that the image of $A$ under the coding map is indeed $\mathcal{K}$, and hence uncountable.

If $(\alpha, \beta) \in A \cap \mathcal{U}_{j}$ then $G(\alpha, \beta)$ can be interpreted symbolically as the substitution $\chi_{j}$ in the following sense: Let $T$ be the ITM and $F$ be the first return map to the interval $[1-\alpha, 1)$.

- The left branch of $F$ is identical to the middle branch of $T$. We denote this symbolically as $1 \rightarrow 2$.

- Assuming that $\frac{1}{k+1}<\alpha \leq \frac{1}{k}$ we get that the middle branch of $F$ involves one iterate of the third branch of $T$ followed by $k$ applications of the left branch of $T: 2 \rightarrow 31^{k}$.

- Still assuming that $\frac{1}{k+1}<\alpha \leq \frac{1}{k}$ we get that the middle branch of $F$ involves one iterate of the third branch of $T$ followed by $k-1$ applications of the left branch of $T: 3 \rightarrow 31^{k-1}$. 
Take $k_{n}=j$ if $G^{n}((\alpha, \beta)) \in \mathcal{U}_{j}$. Let $\Delta_{n}$ be the $n$-th inducing interval, and assume we want to code orbits of the first return map $T_{\Delta_{n}}$ according to the natural partition into branch domains, using symbols $\{1,2,3\}$. Following the above pattern, we see that if $x \in \Delta_{n}$ has itinerary $u$ for $T_{\Delta_{n}}$, then $\chi_{k_{n-1}}(u)$ is the itinerary of $x$ for $T_{\Delta_{n-1}}$. By induction, the limit

$$
s=s_{0} s_{1} s_{2} \cdots=\lim _{i \rightarrow \infty} \chi_{k_{0}} \circ \cdots \circ \chi_{k_{i}}(3)
$$

gives the itinerary of the point 1 . Recall that, since $1 \notin[0,1)$, we defined $T(1)=\lim _{x \rightarrow 1^{-}} T(x)$. Thus $T_{\alpha, \beta}^{i-1}(T(1)) \in B_{j}$ if and only if $s_{i}=j$. Let $\Sigma_{\alpha, \beta}=\overline{\left\{\sigma^{i}(s): i \geq 0\right\}}$ be the corresponding shift space. Then the above coding extends to a map $h: \bar{\Omega} \rightarrow \Sigma_{\alpha, \beta}$ which is continuous and one-to-one, except possibly at the countable set $\cup_{i} T_{\alpha, \beta}^{-i}(\{1-\alpha, 1-\beta\})$.

Proof of Proposition 8. The map $\left.G\right|_{A}$ is coded by the full shift on a countable alphabet. Thus there are countably periodic codes. Since $\left.G\right|_{A}$ is non-uniformly expanding, see Proposition 15, each periodic code corresponds to a single point and the periodic points lie dense in $A$.

We turn to the self-similarity assertion. Let $\left(\alpha_{i}, \beta_{i}\right)=G^{i}(\alpha, \beta)$. Let $\Delta_{n}:=$ $\left[1-\prod_{i=0}^{n-1} \alpha_{i}, 1\right)$. The map $G$ rescales the induced map to be defined on a interval of length 1 , thus $T$ and $T_{\Delta_{n}}$ differ only by scaling for all $k \in \mathbb{N}$ if and only if $(\alpha, \beta)$ is a $G$-periodic point with period $n$.

Proof of Proposition 9. Let $O_{k}$ be the set of points $(\alpha, \beta) \in A$ such that $G^{i}((\alpha, \beta)) \notin \cup_{j>k} \mathcal{U}_{j}$ for all $i \geq 0$. The set $O_{k}$ is a subshift of finite type (in fact, the full shift on $k$ symbols), thus it is uncountable. Clearly the set $\cup_{k} O_{k}$ is dense in $A$. By Theorem 7, for $(\alpha, \beta) \in O_{k}, T_{\alpha, \beta}$ is isomorphic to the shift space $\Sigma_{\alpha, \beta}$. The substitutions $\chi_{k}$ are primitive for all $k \geq 2$. By assumption, $(1,1)$ is not an accumulation point of $\operatorname{orb}(\alpha, \beta)$, so the substitution $\chi_{1}$ appears only in blocks of bounded length, and each "block" $\chi_{1}^{n} \circ \chi_{r}$, $2 \leq r \leq k$, is primitive. Hence $\Sigma_{\alpha, \beta}$ is adic. Note that it is uniquely ergodic as well, but this holds more generally, see Theorem 12 .

\section{Dimension Results}

Proof of Theorem 10. We will compute upper bounds for the box dimension by finding suitable covers of $\bar{\Omega}$. Let $\left(\alpha_{i}, \beta_{i}\right)=G^{i}(\alpha, \beta)$ for $i \geq 0$. The map $G^{i}$ computes the parameters of the ITM that results from the first return map $T_{\Delta_{i}}$ to the interval $\Delta_{i}:=\left[1-\pi_{i}, 1\right)$ where $\pi_{i}:=\prod_{j=0}^{i-1} \alpha_{j}$, so $\pi_{0}=1$. The map $T_{\Delta_{i}}$ consists of three branches, the left branch of $T_{\Delta_{i}}$ is defined on an interval of length $\pi_{i, 1}=\pi_{i}\left(1-\alpha_{i}\right)$, the middle branch is defined on an interval of length $\pi_{i, 2}=\pi_{i}\left(\alpha_{i}-\beta_{i}\right)$, while the length of the right branch is $\pi_{i} \beta_{i}$. Also put $\pi_{i, 3}=\pi_{i} \alpha_{i}$. Let $k_{i}=\left\lfloor\frac{1}{\alpha_{i}}\right\rfloor$. Then the intersection of $\bar{\Omega}$ and the domain of the left branch can be covered by $k_{i}-1$ intervals of length $\pi_{i} \beta_{i}=\pi_{i+1,3}$ and one interval of length $\pi_{i}\left(\beta_{i}-\alpha_{i} \beta_{i+1}\right)=\pi_{i+1,2}$. The 
domain of the middle branch is an interval of length $\pi_{i}\left(\alpha_{i}-\beta_{i}\right)=\pi_{i+1,1}$ and the interval $\Delta_{i+1}$ is the union an interval of length $\pi_{i}\left(\alpha_{i}-\beta_{i}\right)=\pi_{i+1,1}$ and an interval of length $\pi_{i} \beta_{i}=\pi_{i+1,3}$. This is illustrated in Figure 1 .

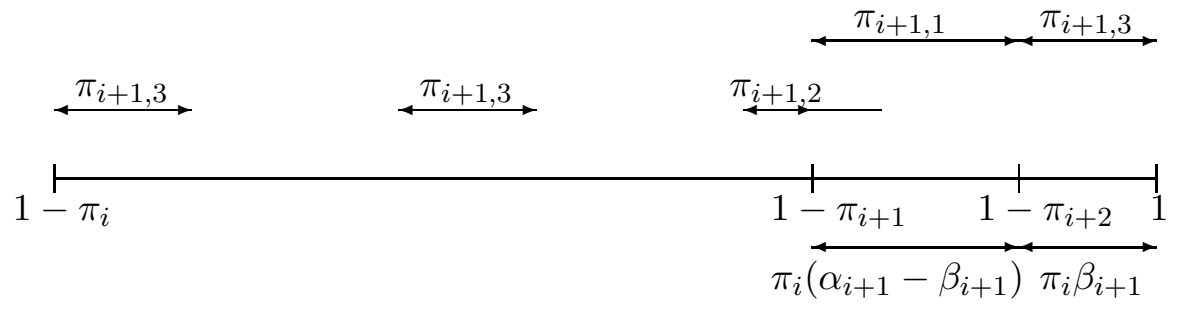

Figure 4. Cover of $\left[1-\pi_{i}\right) \cap \bar{\Omega}$ with intervals $\pi_{i+1, j}$ for $k_{i+1}=3$.

The map $T$ pushes the interval $\Delta_{i}$ and its subintervals of length $\pi_{i, j}$ around through $[0,1)$. The first return map to any image $T^{k}\left(\Delta_{i}\right)$ has the same structure as $T_{\Delta_{i}}$, and hence $T^{k}\left(\Delta_{i}\right) \cap \bar{\Omega}$ is covered by the same number of intervals of length $\pi_{i, j}$ as $\Delta_{i}$. Similarly, any interval of length $\pi_{i, j}$ can be replaced by a number of intervals of length $\pi_{i+1, j^{\prime}}$, according to the scheme:

$$
\begin{array}{lll}
\pi_{i, 1} & \text { is covered by } & 1 \times \pi_{i+1,2} \text { and }\left(k_{i+1}-1\right) \times \pi_{i+1,3} ; \\
\pi_{i, 2} & \text { is covered by } & 1 \times \pi_{i+1,1} ; \\
\pi_{i, 3} & \text { is covered by } & 1 \times \pi_{i+1,1} \text { and } 1 \times \pi_{i+1,3} .
\end{array}
$$

If we denote the number of such intervals by $l_{i, j}$, then the computation of their increase can be performed using a matrix:

$$
\left(\begin{array}{c}
l_{i+1,1} \\
l_{i+1,2} \\
l_{i+1,3}
\end{array}\right)=\left(\begin{array}{ccc}
0 & 1 & 1 \\
1 & 0 & 0 \\
k_{i+1}-1 & 0 & 1
\end{array}\right)\left(\begin{array}{l}
l_{i, 1} \\
l_{i, 2} \\
l_{i, 3}
\end{array}\right) .
$$

The upper box dimension of $\bar{\Omega}$ is bounded by

$$
\overline{\operatorname{dim}_{\mathrm{B}}}(\bar{\Omega}) \leq \limsup _{i} \frac{\log \left(l_{i, 1}+l_{i, 2}+l_{i, 3}\right)}{-\log \pi_{i}} .
$$

Let $\rho=\log \left(\bar{r}_{2}\right) / \log 2=0.84955 \cdots$, where $\bar{r}_{2}$ is the leading root of $P_{2}$ defined by Equation (2). One can verify that $\bar{r}_{k} \leq k^{\rho}$ for all $k \geq 1$. Therefore we get for arbitrary $(\alpha, \beta) \in A$ :

$$
\begin{aligned}
\overline{\operatorname{dim}}_{\mathrm{B}}\left(\bar{\Omega}_{\alpha, \beta}\right) & \leq \limsup _{i} \frac{\log \left(l_{i, 1}+l_{i, 2}+l_{i, 3}\right)}{-\log \pi_{i}} \\
& \leq \limsup _{i} \frac{\log \left(C \prod_{j=0}^{i} \bar{r}_{k_{j}}\right)}{\log \prod_{j=0}^{i} k_{j}} \\
& =\limsup _{i} \frac{\sum_{j=0}^{i} \log \bar{r}_{k_{j}}}{\sum_{j=0}^{i} \log k_{j}} \leq \rho .
\end{aligned}
$$

This proves the upper bound. 
As an example, if $(\alpha, \beta)$ is the fixed point of $G$ in $\mathcal{U}_{k}, k \geq 2$, then $\beta=$ $\alpha^{2}$ and $\alpha$ is the root $r_{k} \in\left(\frac{1}{k+1}, \frac{1}{k}\right]$ of the polynomial $P_{k}$ from (2). The characteristic polynomial of the matrix in (同) is also $P_{k}$ for $k=k_{i}$; recall that $\bar{r}_{k}$ is its leading root. Then we find $l_{i, j}=\mathcal{O}\left(\bar{r}_{k}^{i}\right)$ for each $j$, and therefore $\overline{\operatorname{dim}_{\mathrm{B}}}\left(\bar{\Omega}_{\alpha, \beta}\right) \leq-\log \left(\bar{r}_{k}\right) / \log \left(r_{k}\right)$. For $k=3$ this bound equals $0.6635 \cdots$ and in BK (due to self-similarity) it is shown that if $k_{i} \equiv 3$, it is exact and equal to the Hausdorff dimension. The same can be shown if $k_{i} \equiv k$ is constant. If $k$ is taken very large, then $\bar{r}_{k} \approx \sqrt{k}+\frac{1}{2}$, while $\alpha=r_{k} \approx \frac{1}{k}$. Therefore $\frac{1}{2} \leq \overline{\operatorname{dim}_{\mathrm{B}}}\left(\bar{\Omega}_{\alpha, \beta}\right) \leq \frac{1}{2}+\epsilon$ for sufficiently large $k$. This is in agreement with the lower bound given in Bo1].

Now to prove that $\operatorname{dim}_{\mathrm{H}}\left(\bar{\Omega}_{\alpha, \beta}\right)=0$ for some values of $(\alpha, \beta)$ we argue as follows: Let as before $\pi_{i}$ be the length of the domain of the $i$-th first return map $T_{\Delta_{i}}$. We can cover $\bar{\Omega}$ with $N_{i}:=l_{i, 1}+l_{i, 2}+l_{i, 3}$ intervals of length $\leq \pi_{i}$. Given $N_{i}$ and $\pi_{i}$, choose $\alpha_{i}$ and $\beta_{i}$ (thus determining $\beta_{i+1}$ ) so that

$$
N_{i}\left\lfloor\frac{1}{\alpha_{i}}+1\right\rfloor\left(\pi_{i} \beta_{i}\right)^{1 / i}+N_{i}\left\lfloor\frac{\alpha_{i}}{\beta_{i}}\right\rfloor\left(\pi_{i} \alpha_{i} \beta_{i+1}\right)^{1 / i}<1 .
$$

This can be achieved as follows: write $\alpha_{i}=\frac{1}{k_{i}}-\varepsilon$ and $\beta_{i}=k_{i} \varepsilon+\varepsilon^{\prime}$ for $0<\varepsilon^{\prime} \ll \varepsilon \ll\left(k_{i}\right)^{-2}$ and $0<\varepsilon^{\prime}<\alpha_{i}-k_{i} \varepsilon$. Then (6) follows from

$$
N_{i} \pi_{i}^{1 / i}\left(k_{i}+1\right)^{1+1 / i} \varepsilon^{1 / i}+N_{i} \pi_{i+1}^{1 / i} \frac{1}{k_{i}^{2} \varepsilon}\left(\varepsilon^{\prime}\right)^{1 / i}<1,
$$

which is easily satisfied for $\left(\varepsilon, \varepsilon^{\prime}\right)$ taken in an appropriate region near $(0,0)$.

By the above reasoning, the domain of the left branch of $T_{\Delta_{i}}$ can be covered by $\left\lfloor\frac{1}{\alpha_{i}}\right\rfloor$ intervals of length $\pi_{i} \beta_{i}$. The domain of the middle branch of $T_{\Delta_{i}}$ is the domain of the left branch of $T_{\Delta_{i+1}}$ and can therefore be covered by $\left\lfloor\frac{1}{\alpha_{i+1}}\right\rfloor=\left\lfloor\frac{\alpha_{i}}{\beta_{i}}\right\rfloor$ intervals of length $\pi_{i} \alpha_{i} \beta_{i+1}$. Finally, the domain of the right branch of $T_{\Delta_{i}}$ is a single interval of length $\pi_{i} \beta_{i}$. Putting these things together, we derive that $\bar{\Omega}$ is covered by $N_{i}\left\lfloor\frac{1}{\alpha_{i}}+1\right\rfloor$ intervals of length $\pi_{i} \beta_{i}$ and $N_{i}\left\lfloor\frac{\alpha_{i}}{\beta_{i}}\right\rfloor$ intervals of length $\pi_{i} \alpha_{i} \beta_{i+1}$. Due to the choice (6), the corresponding "critical exponent" of this cover is $\leq 1 / i$. If $(\alpha, \beta)$ is such that indeed (6) holds for infinitely many $i$, then $\operatorname{dim}_{H}\left(\bar{\Omega}_{\alpha, \beta}\right)=\underline{\operatorname{dim}_{\mathrm{B}}}\left(\Omega_{\alpha, \beta}\right)=0$.

In particular, if $\left(\alpha_{i}, \beta_{i}\right)$ is a sequence which alternates values satisfying (6) with long blocks of $k_{i}:=\left\lfloor 1 / \alpha_{i}\right\rfloor \equiv 3$, say, then we will find that the upper box dimension $\overline{\operatorname{dim}_{\mathrm{B}}}\left(\bar{\Omega}_{\alpha, \beta}\right)=0.6635 \ldots$ is larger than the Hausdorff dimension $\operatorname{dim}_{\mathrm{H}}\left(\bar{\Omega}_{\alpha, \beta}\right)=0$. This concludes the proof.

\section{InVARIANT Measures}

In this section we prove Theorems 2, 11 and 12 as well as some related results. Throughout the section, $T_{\alpha, \beta}$ is assumed to be of type infinity, with corresponding sequence $\left(k_{i}\right)$. Let us start with some notation. Let 
$s=s_{0} s_{1} s_{2} s_{3} \ldots$ be the fixed point of $\chi_{k_{0}} \circ \chi_{k_{1}} \circ \ldots$ For $a \in\{1,2,3\}$, write

$$
\operatorname{freq}_{a}\left(T_{\alpha, \beta}\right)=\bigcap_{N \geq 1} \mathrm{CH}_{n \geq N, k \geq 1} \frac{1}{n} \#\left\{k \leq i<k+n ; s_{i}=a\right\},
$$

where $\mathrm{CH}$ denotes the convex hull over all $k \geq 1$ and $n \geq N$. Without the subscript $a$, freq $\left(T_{\alpha, \beta}\right)$ is the vector with three components. We say that freq $\left(T_{\alpha, \beta}\right)$ exists if each component is a single point, which then satisfies $\operatorname{freq}_{1}\left(T_{\alpha, \beta}\right)+\operatorname{freq}_{2}\left(T_{\alpha, \beta}\right)+\operatorname{freq}_{3}\left(T_{\alpha, \beta}\right)=1$.

Lemma 16. If $(\alpha, \beta) \neq(\hat{\alpha}, \hat{\beta})$, then $\operatorname{freq}\left(T_{\alpha, \beta}\right) \cap \operatorname{freq}\left(T_{\hat{\alpha}, \hat{\beta}}\right)=\emptyset$.

In other words, the frequency vector freq uniquely determines the parameter $(\alpha, \beta)$.

Proof. Each substitution $\chi_{k}$ has an associated matrix $A_{k}$ whose characteristic polynomial is $P_{k}$ as in (2). Define the simplex $S=\left\{(x, y, z) \in \mathbb{R}^{3}: 0 \leq\right.$ $x, y, z, x+y+z=1\}$; the coordinates $x, y, z$ will play the role of $\operatorname{freq}_{1}\left(T_{\alpha, \beta}\right)$, freq $_{2}\left(T_{\alpha, \beta}\right)$, resp. freq ${ }_{3}\left(T_{\alpha, \beta}\right)$. The matrix $A_{k}$ gives rise to a mapping $F_{k}$ on $S$ given by

$$
F_{k}(x, y, z)=\frac{1}{k(y+z)+x+y}(k(y+z)-z, x, y+z) .
$$

Pass to new coordinates $\xi=x+y$ and $\eta=y+z$. Since $x+y+z=1$ on $S$, this gives a new map

$$
\tilde{F}_{k}(\xi, \eta)=\left(1-\frac{\eta}{k \eta+\xi}, \frac{1}{k \eta+\xi}\right),
$$

defined on the triangle $\tilde{S}=\{(\xi, \eta): \xi, \eta \leq 1,1 \leq \xi+\eta\}$. The map $\tilde{F}_{k}$ preserves lines, and the images $\tilde{F}_{k}(\tilde{S})$ are triangles $\mathcal{V}_{k}$ with corners $(1,1)$, $\left(\frac{k-1}{k}, \frac{1}{k}\right)$ and $\left(\frac{k}{k+1}, \frac{1}{k+1}\right)$. These triangles have disjoint interiors and tile the triangle $\tilde{S}$. On $\mathcal{V}_{k}$, there is one inverse map

$$
\tilde{F}_{k}^{-1}(\xi, \eta)=\frac{1}{\eta}(1+k(\xi-1), 1-\xi) .
$$

The map $\tilde{F}_{k}$ has derivative

$$
D \tilde{F}_{k}(\xi, \eta)=\frac{1}{(k \eta+\xi)^{2}}\left(\begin{array}{cc}
\eta & -\xi \\
-1 & -k
\end{array}\right)
$$

eigenvalues $\lambda_{ \pm}=\frac{1}{2(k \eta+\xi)^{2}}\left(k-\eta \pm \sqrt{(k+\eta)^{2}+4 \xi}\right)$, and determinant $\frac{-1}{(k \eta+\xi)^{3}}$. This determinant is less than or equal to 1 in absolute value. Write

$$
Z_{i, j}=\tilde{F}_{k_{j}} \circ \tilde{F}_{k_{j+1}} \circ \cdots \circ \tilde{F}_{k_{i}}(\tilde{S}) \text { and } Z_{j}=\lim _{i \rightarrow \infty} Z_{i, j} .
$$

Since $\tilde{F}_{k}$ preserves lines but contracts area, $Z_{i, j}$ is convex, while $Z_{j}$ is a point or a straight arc.

Since $(\alpha, \beta) \neq(\hat{\alpha}, \hat{\beta})$ and $G^{2}$ is (non-uniformly) expanding, see Proposition 15, $\left(k_{i}\right)$ and $\left(\hat{k}_{i}\right)$ are not the same. Therefore $Z_{i, 0}\left(T_{\alpha, \beta}\right)$ and $Z_{i, 0}\left(T_{\hat{\alpha}, \hat{\beta}}\right)$ are not the same for some $i$. If they are disjoint, then also $Z_{0}\left(T_{\alpha, \beta}\right) \neq$ $Z_{0}\left(T_{\hat{\alpha}, \hat{\beta}}\right)$. Transforming back to the coordinates $x, y, z$, we obtain that the frequency vectors $\operatorname{freq}\left(T_{\alpha, \beta}\right) \neq \operatorname{freq}\left(T_{\hat{\alpha}, \hat{\beta}}\right)$. 
If $Z_{i, 0}\left(T_{\alpha, \beta}\right)$ and $Z_{i, 0}\left(T_{\hat{\alpha}, \hat{\beta}}\right)$ meet in their boundaries for all $i \geq 1$, then $k_{j}=\hat{k}_{j}=1$ for all $i \geq i_{0}$. Note that $\tilde{F}_{1}$, under iteration, contracts the triangle $\tilde{S}$ to the left upper corner $(0,1)$. Therefore $Z_{i_{0}}\left(T_{\alpha, \beta}\right)=Z_{i_{0}}\left(T_{\hat{\alpha}, \hat{\beta}}\right)=(0,1)$. Since $k_{i} \neq \hat{k}_{i}$ for some $i<i_{0}$, we get that $Z_{0}\left(T_{\alpha, \beta}\right) \neq Z_{0}\left(T_{\hat{\alpha}, \hat{\beta}}\right)$ after all.

Lemma 17. Let $T_{\alpha, \beta}$ be of infinite type. Then $Z_{0}\left(T_{\alpha, \beta}\right)$ is a single point if and only if $T_{\alpha, \beta}$ is uniquely ergodic.

It is clear that freq $\left(T_{\alpha, \beta}\right)$ exists if and only if $Z_{0}\left(T_{\alpha, \beta}\right)$ is a single point. Moreover, $Z_{0}\left(T_{\alpha, \beta}\right)$ is a single point if and only if $Z_{i}\left(T_{\alpha, \beta}\right)$ is a single point for some $i \geq 0$.

Proof. By Theorem 7, $\left(\Omega, T_{\alpha, \beta}\right)$ is isomorphic to a shift space $\Sigma_{\alpha, \beta}$ via an "isomorphism" which is one-to-one except on the countable set $\cup_{i} T_{\alpha, \beta}^{-i}(\{1-$ $\alpha, 1-\beta\})$. This set supports no invariant probability measure. So it suffices to determine when $\left(\Sigma_{\alpha, \beta}, \sigma\right)$ is uniquely ergodic.

First assume that $Z_{0}$ is a singleton. Let $\varepsilon>0$ be arbitrary and $C=c_{0} \ldots c_{M}$ be any word. Each $t \in \Sigma_{\alpha, \beta}$ is a concatenation of words of the form $W(i)=$ $\chi_{k_{0}} \circ \cdots \circ \chi_{k_{N}}(i)$, for $i \in\{1,2,3\}$ and some fixed $N$. More precisely,

$$
t=W W\left(i_{1}\right) W\left(i_{2}\right) W\left(i_{3}\right) \ldots
$$

where $W$ is a suffix (possibly empty) of $W\left(i_{0}\right)$. Let us say that an occurrence of $C$ in $t N$-overlaps if $t_{[k, k+M]}=C$ and $k<\left|W W\left(i_{1}\right) \ldots W\left(i_{r}\right)\right|<k+M$ for some $r$. By taking $N$ sufficiently large, we can assume that

$$
\limsup _{n} \frac{1}{n} \#\left\{i ; C=t_{[i, i+M]} \text { and } N \text {-overlaps }\right\} \leq \varepsilon,
$$

uniformly over all $t \in \Sigma_{\alpha, \beta}$. Therefore

$$
\lim _{n} \frac{1}{n} \#\left\{0 \leq i<n: C=t_{[i, i+M]} \text { and does not } N \text {-overlap }\right\}
$$

differs from $v(C, t):=\lim _{n} \frac{1}{n} \#\left\{0 \leq i<n ; C=t_{[i, i+M]}\right\}$ by no more than $\varepsilon$.

Since $Z_{0}\left(T_{\alpha, \beta}\right)$ is a singleton, there exists freq $\in \tilde{S}$, such that for each $\varepsilon^{\prime}>0$ there is $M>N$ such that for each word $V(b)=\chi_{k_{N+1}} \circ \cdots \circ \chi_{k_{M}}(b)$,

$$
\mid \frac{\#\left\{0<j \leq|V(b)| ; V(b)_{j}=a\right\}}{|V(b)|}-\text { freq }_{a} \mid<\varepsilon^{\prime},
$$

for $a, b=1,2,3$. For each $a \in\{1,2,3\}$, let $C_{a}$ be the number of occurrences of $C$ in the word $W(a)$. Then

$$
v(C, t)=\frac{\sum_{a=1}^{3} C_{a} \cdot\left(\text { freq }_{a}+\mathcal{O}\left(\varepsilon^{\prime}\right)\right)}{\sum_{a=1}^{3}|W(a)|\left(\text { freq }_{a}+\mathcal{O}\left(\varepsilon^{\prime}\right)\right)}+\mathcal{O}(\varepsilon) .
$$

Since $\varepsilon$ and $\varepsilon^{\prime}$ are arbitrary, we see that $v(C, t)$ is independent of the string $t$. Thus unique ergodicity follows.

Conversely, if $Z_{0}\left(T_{\alpha, \beta}\right)$ is not a single point, it has diameter $\operatorname{diam} Z_{0}:=\delta>$ 0 . It follows that diam $Z_{i, 0}\left(T_{\alpha, \beta}\right) \geq \delta$ for every $i \in \mathbb{N}$. The extremal points of $Z_{i, 0}$ are the images under $\tilde{F}_{k_{0}} \circ \cdots \circ \tilde{F}_{k_{i}}$ of the corners of $\tilde{S}$. For simplicity, we 
can assume that the vertical height of $Z_{i, 0} \geq \delta / 2$. Recall that the variables $x, y$ and $z$ give the frequencies of the symbols 1,2 and 3 in words of $\Sigma_{\alpha, \beta}$, and that $x=1-\eta$.

There exists $a, b \in\{1,2,3\}$ such that for infinitely many $j$, we find that the frequencies of the symbol 1 :

$$
\frac{1}{\left|\chi_{k_{0}} \circ \chi_{k_{1}} \circ \cdots \circ \chi_{k_{j}}(a)\right|} \#\left\{\text { symbols } 1 \text { in } \chi_{k_{0}} \circ \chi_{k_{1}} \circ \ldots \chi_{k_{i}}(a)\right\}
$$

and

$$
\frac{1}{\left|\chi_{k_{0}} \circ \chi_{k_{1}} \circ \cdots \circ \chi_{k_{i}}(b)\right|} \#\left\{\text { symbols } b \text { in } \chi_{k_{0}} \circ \chi_{k_{1}} \circ \ldots \chi_{k_{i}}(b)\right\}
$$

differ by at least $\delta / 2$. Hence $\Sigma_{\alpha, \beta}$ is not uniquely ergodic.

Proof of Theorem 11. According to Lemma 17 we need to show that compositions $\tilde{F}_{k_{0}} \circ \tilde{F}_{k_{1}} \circ \ldots$ do not contract the simplex $\tilde{S}$ to a single point. The composition of two maps $\tilde{F}$ has the form

$$
\tilde{F}^{2}(\xi, \eta)=\tilde{F}_{k^{\prime}} \circ F_{k}(\xi, \eta)=\left(1-\frac{1}{k^{\prime}+(k-1) \eta+\xi}, \frac{k \eta+\xi}{\left.k^{\prime}+(k-1) \eta\right)+\xi}\right) .
$$

The map $\tilde{F}_{k_{2 i-1}} \circ \tilde{F}_{k_{2 i}}$ acts on the second component as

$$
h_{i}: \eta \mapsto \frac{k_{2 i} \eta+\xi}{k_{2 i-1}+\left(k_{2 i}-1\right) \eta+\xi} .
$$

We will show $\lim _{j \rightarrow \infty} h_{1} \circ h_{2} \circ \cdots \circ h_{j}([0,1])$ is a non-degenerate interval.

Let $\tau=1 /(\lambda-1)$ and $\tau^{\prime}=1-1 / \lambda$. Take $i_{0}$ so large that $k_{i} \geq \lambda k_{i-1}$ for all $i \geq i_{0}$ as well as $\tau<\tau^{\prime} k_{2 i_{0}-2}$. Obviously, $h_{i}$ is an increasing Möbius transformation on $[0,1]$. Assume that $2 i \geq i_{0}+2$. Then

$$
h_{i}\left(\tau^{\prime}\right) \geq \frac{\tau^{\prime} k_{2 i}}{k_{2 i} / \lambda+\tau^{\prime}\left(k_{2 i}-1\right)+1} \geq \frac{\tau^{\prime}}{1 / \lambda+\tau^{\prime}}=\tau^{\prime} .
$$

It follows that $h_{i}\left(\left[\tau^{\prime}, 1\right]\right) \subset\left(\tau^{\prime}, 1\right)$, and hence

$$
\lim _{j \rightarrow \infty} h_{i_{0}} \circ h_{i_{0}+1} \circ \cdots \circ h_{j}(1) \geq \tau^{\prime} .
$$

On the other hand, if $\eta \leq \tau / k_{2 i}$, then $h_{i}(\eta) \leq \frac{1+\tau}{k_{2 i-1}} \leq \frac{1+\tau}{\lambda k_{2 i-2}}=\frac{\tau}{k_{2 i-2}}$. By induction we find $\lim _{j \rightarrow \infty} h_{i_{0}} \circ h_{i_{0}+1} \circ \cdots \circ h_{j}(0) \leq \frac{\tau}{k_{2 i_{0}-2}}$. Therefore

$$
\begin{aligned}
{[p, q] } & :=\lim _{j \rightarrow \infty} h_{1} \circ h_{2} \circ \cdots \circ h_{j}([0,1]) \\
& =h_{1} \circ h_{2} \circ \cdots \circ h_{i_{0}-1}\left(\lim _{j \rightarrow \infty} h_{i_{0}} \circ h_{i_{0}+1} \circ \cdots \circ h_{j}([0,1])\right) \\
& \supset h_{1} \circ h_{2} \circ \cdots \circ h_{i_{0}-1}\left(\left[\frac{\tau}{k_{2 i_{0}-2}}, \tau^{\prime}\right]\right) .
\end{aligned}
$$

In particular, $p<q$. Lemma 17 implies that $\Sigma_{\alpha, \beta}$ and therefore $T_{\alpha, \beta}$ are not uniquely ergodic.

Proof of Theorem 12. According to Lemma 17, we need to show that $Z_{0}$ (or equivalently $Z_{1}$ ) is a single point. The first component of $\tilde{F}^{2}(\xi, \eta)$ 
contracts the interval to a single point because the derivative with respect to $\xi$ is $\leq 1$ with equality only if $k=k^{\prime}=1$ and $\xi=0$. Hence $Z_{0}$ has "width" 0. For the "height", we need the second component of $\tilde{F}^{2}(\xi, \eta)$, for which we will use the maps $h_{i}$ from the proof of Theorem 11. The compositions $H_{i}=h_{1} \circ \cdots \circ h_{i}$ are Möbius transformations represented by the matrix-multiplications

$$
\begin{aligned}
B_{i} & =\left(\begin{array}{cc}
b_{1,1}^{i} & b_{1,2}^{i} \\
b_{2,1}^{i} & b_{2,2}^{i}
\end{array}\right) \\
& =\left(\begin{array}{cc}
k_{2} & \xi_{1} \\
k_{2}-1 & k_{1}+\xi_{1}
\end{array}\right) \cdots\left(\begin{array}{cc}
k_{2 i} & \xi_{i} \\
k_{2 i}-1 & k_{2 i-1}+\xi_{i}
\end{array}\right),
\end{aligned}
$$

where the $\xi_{i} \in[0,1]$. Since

$$
H_{i}([0,1]) \subset\left[H_{i}(0), \lim _{\eta \rightarrow \infty} H_{i}(\eta)\right]=\left[b_{1,2}^{i} / b_{2,2}^{i}, b_{1,1}^{i} / b_{2,1}^{i}\right],
$$

it suffices to show that $B_{i}$ contracts the cone $\mathcal{C}:=\left(\mathbb{R}_{\geq 0}\right)^{2}$ to a one-dimensional subcone as $i \rightarrow \infty$. In order to do this, we use Hilbert metric on $\mathcal{C}$ : Given $v, w \in \mathcal{C}$, define

$$
\Theta(v, w)=\log \left(\frac{\inf \{\mu ; \mu v-w \in \mathcal{C}\}}{\sup \{\lambda ; w-\lambda v \in \mathcal{C}\}}\right) .
$$

In fact, $\Theta$ is a semi-metric, because $\Theta(v, w)=0$ if and only if $v$ is a multiple of $w$. Let $T: \mathcal{C} \rightarrow \mathcal{C}$ be a linear map. It is shown in e.g. [B] that $\Theta(T(v), T(w)) \leq \tanh (D / 4) \Theta(v, w)$ for $D=\sup _{v^{\prime}, w^{\prime} \in T(\mathcal{C})} \Theta\left(v^{\prime}, w^{\prime}\right)$. In particular, $T$ is a contraction if $T$ maps $\partial \mathcal{C} \backslash\{0\}$ into the interior of $\mathcal{C}$.

In our setting, the transformations $T$ are represented by matrices of the form $\left(\begin{array}{cc}k & \xi \\ k-1 & k^{\prime}+\xi\end{array}\right)$, and we can easily check that $D$ is assumed by taking $v^{\prime}=(1,0)^{\top}$ and $w^{\prime}=(0,1)^{\top}$, so $D=\log \frac{k\left(k^{\prime}+\xi\right)}{(k-1) \xi}$. Hence the contraction factor is

$$
\begin{aligned}
\tanh \left(\frac{D}{4}\right) & =\frac{\sqrt[4]{\frac{k\left(k^{\prime}+\xi\right)}{(k-1) \xi}}-\sqrt[4]{\frac{(k-1) \xi}{k\left(k^{\prime}+\xi\right)}}}{\sqrt[4]{\frac{k\left(k^{\prime}+\xi\right)}{(k-1) \xi}}+\sqrt[4]{\frac{(k-1) \xi}{k\left(k^{\prime}+\xi\right)}}} \\
& =\frac{\sqrt{k\left(k^{\prime}+\xi\right)}-\sqrt{(k-1) \xi}}{\sqrt{k\left(k^{\prime}+\xi\right)}+\sqrt{(k-1) \xi}} \\
& =1-2 \frac{\sqrt{k(k-1)\left(k^{\prime}+\xi\right) \xi}-(k-1) \xi / 2}{k k^{\prime}+\xi} \\
& \leq 1-4 \frac{k-1}{k} \sqrt{\frac{\xi}{k^{\prime}}} .
\end{aligned}
$$

The variable $\xi$ is the result of iterating $\tilde{h}: \xi \mapsto 1-\frac{1}{k^{\prime}+\xi+(k-1) \eta}$, the first component of $\tilde{F}^{2}$. The image $\tilde{h}(\xi) \geq \frac{1}{2}$ unless $k=k^{\prime}=1$. If $k=k^{\prime}=1$, then $\xi=0$ is an indifferent attracting fixed point, and $\tilde{h}^{n}(1)=1 /(n+1)$. Therefore, in the above calculation, we can estimate $\xi \geq 1 / L_{2 i}$. Hence, the height of $Z_{0}$ is less than $\prod_{i} \tanh \left(D_{i} / 4\right) \leq \exp \left(-4 \sum_{i} \frac{k_{2 i}-1}{k_{2 i}} \sqrt{\frac{1}{L_{2 i} k_{2 i-1}}}\right)$. The 
assumption on $\left(k_{i}\right)$ gives that $Z_{0}$ is indeed a single point. This finishes the proof of the theorem with condition 3 .

For condition (舟), as in the proof of the part with condition (3), the width of the $Z_{1}$ is 0 . The compositions of Möbius transformations $H_{i}=h_{1} \circ h_{2} \circ \cdots \circ h_{i}$ satisfy,

$$
\left|H_{i}([0,1])\right|=\sqrt{H_{i}^{\prime}(0) \cdot H_{i}^{\prime}(1)} \leq H_{i}^{\prime}(0) \leq \prod_{j=1}^{i} h_{j}^{\prime}(0),
$$

where the last inequality follows because each $h_{i}$ is increasing with decreasing derivative. We compute $h_{j}^{\prime}(\eta)=\frac{k_{2 j} k_{2 j-1}+\xi}{\left(k_{2 j-1}+\left(k_{2 j}-1\right) \eta+\xi\right)^{2}}$, and therefore $h_{j}^{\prime}(0) \leq$ $k_{2 j} /\left(k_{2 j-1}+1 / L_{2 j}\right)$. It follows that

$$
\operatorname{diam} Z_{1} \leq \lim _{j} H_{i}([0,1]) \leq \lim _{i} \prod_{1 \leq j \leq i} \frac{k_{2 j}}{k_{2 j-1}+\frac{1}{L_{2 i}}}=0 .
$$

Lemma 17 yields unique ergodicity.

Proof of Corollary 13. Note that the coding map $(\alpha, \beta) \mapsto k \in \mathcal{K}$ is continuous on $A$. Hence it suffices to consider the space $\mathcal{K}$. For any cylinder $C_{e_{1} \ldots e_{n}}=\left\{k \in \mathcal{K} \mid k_{i}=e_{i}, i=1, \ldots n\right\}$, let $U_{e_{1} \ldots e_{n}}^{m}=\left\{k \in \mathcal{K} \mid k_{i}=e_{i}, i=\right.$ $\left.1, \ldots n, k_{n+i}=2, i=1, \ldots m\right\}$. Clearly $\mathcal{U}^{m}=\cup_{n} \cup_{e_{1} \ldots e_{n}} U_{e_{1} \ldots e_{n}}^{m}$ is open and dense in $\mathcal{K}$. Moreover, for each $k \in U_{e_{1} \ldots e_{n}}^{m}$ we have

$$
\sum_{i} \frac{k_{2 i}-1}{k_{2 i}} \sqrt{\frac{1}{k_{2 i-1} L_{2 i}}} \geq \frac{m-2}{4 \sqrt{2}} .
$$

Therefore $\cap_{m} \mathcal{U}^{m}$ is a dense $G_{\delta}$ set of sequence $k$ satisfying Condition (3) This proves the corollary.

Proof of Corollary 14. The maximum of two ergodic measures corresponds to the at most two extremal points of the sets $Z_{0}$ in the proof of Lemma 16, see [K]. This also follows immediately from the result of Buzzi and Hubert, $\mathrm{BH}$.

The next result gives a candidate (modulo finiteness) of an invariant measure.

Proof of Theorem 2. Let $\mathcal{V}$ be a cover of $\Omega$ by the intervals forming $\Omega_{n}$ satisfying $J \cap \Omega \neq \emptyset$. The map $T$ induces a multivalued map $\psi$ of the $J^{\prime}$ 's by $\psi(J)=J^{\prime}$ if $T(J) \cap J^{\prime} \neq \emptyset$. Thus $\psi$ has one value at $J$ if $\left.T\right|_{J}$ is continuous, otherwise it has two values provided $n$ is sufficiently large. As a result, at most $r$ of the $J^{\prime} s$ can have more than one $\psi$-preimages, where $r$ is the number of discontinuity points.

Take $\varepsilon>0$ so small that any two $J, J^{\prime} \in \mathcal{V}$ are at least $\varepsilon$ apart. Let $\mathcal{U} \stackrel{\text { def }}{=}\left\{U_{i}\right\}$ be an open cover of $\Omega$ with the diameters of the $U_{i}$ all less than $\varepsilon$. Suppose $J$ is an interval such $\psi(J)$ has exactly one preimage. If the subcover of $\mathcal{U}$ covering $J$ gives a good approximation of $H_{d}(J)$, i.e., 
$\sum_{U_{i} \cap J \neq \emptyset} \operatorname{diam}\left(U_{i}\right)^{d} \approx H_{d}(J)$, then the translated subcover $\left\{T\left(U_{i}\right)\right\}_{U_{i} \cap J \neq \emptyset}$ satisfies $\sum_{U_{i} \cap J \neq \emptyset} \operatorname{diam}\left(T\left(U_{i}\right)\right)^{d} \approx H_{d}(T(J))$. Since there are only finitely many intervals $J$ such $\psi(J)$ has more than one preimage, the union of these intervals is negligible as the $n \rightarrow \infty$ (and hence $\varepsilon \rightarrow 0$ ). So when minimizing over all $\varepsilon$-covers $\mathcal{U}$, we can restrict ourselves to $T$-invariant $\varepsilon$-covers and find that Hausdorff measure is $T$-invariant.

\section{AcKnowledgements}

We thank Pascal Hubert for useful conversations and the anonymous referee for many valuable suggestions.

\section{REFERENCES}

[AKT] R. Adler, B. Kitchens, C. Tresser, Dynamics of non-ergodic piecewise affine maps of the torus, Ergod. Th. Dyn. Sys. 21 (2001) 959-1000.

[ACP] P. Ashwin, W. Chambers, G. Petrov, Lossless digital flow oscillations; approximation of invariant fractals, Intl. J. Bifurcation and Chaos, 7 (1997) 2603-2610.

[B] G. Birkhoff, Extensions of Jentzsch's theorem, Trans. Amer. Math. Soc. 85 (1957) 219-227.

[Bo] M. Boshernitzan, A unique ergodicity of minimal symbolic flows with linear block growth rate, Journal d'Anal. Math. 44 (1984/5) 77-96.

[Bo1] M. Boshernitzan, Quantatitive recurrence results, Invent. Math. 113 (1993) 617631.

[BK] M. Boshernitzan, I. Kornfeld, Interval translation mappings, Ergod. Th. Dyn. Sys. 15 (1995) 821-831.

[BH] J. Buzzi, P. Hubert, Piecewise monotone maps without periodic points: Rigidity, measures and complexity, IML preprint 2001-27.

[C1] J. Cassaigne, Facteurs spéciaux et complexité, Bull. Belg. Math. Soc. 4 (1997) 67-88.

[C2] J. Cassaigne, Computing the subword complexity of an s-adic sequence: application to a family of interval translation maps, in preparation.

[G] A. Goetz, Dynamics of a piecewise rotation, Discrete Contin. Dynam. Systems 4 (1998) 593-608.

[HR] F. Hofbauer, P. Raith, Topologically transitive subsets of piecewise monotonic maps, which contain no periodic points, Monatsh. Math. 107 (1989) 217-239.

$[\mathrm{KH}]$ A. Katok, B. Hasselblatt, Introduction to the modern theory of dynamical systems, Cambridge Univ. Press (1995).

[K] M. Keane, Non-ergodic interval exchange transformations, Israel J. Math. 26 (1977) 188-196.

[L] G. Levitt, La dynamique des pseudo-groupes de rotations, Invent. Math. 113 (1993) 633-670.

[LV] J. Lowenstein, F. Vivaldi, Embedding dynamics for round-off errors near a periodic orbit, Chaos 10 (2000) 747-755.

[M] H. Masur, Interval exchange transformations and measured foliations, Ann. of Math. 115 (1982) 169-200.

[R] G. Rauzy, Echanges d'intervalles et transformations induites, Acta Arith. 34 (1979) 315-328.

[ST] J. Schmeling, S. Troubetzkoy, Interval translation mappings, in "Dynamical systems from crystals to chaos" J.-M. Gambaudo et al. eds. World Scientific, Singapore (2000) 291-302.

[V1] W. Veech, Gauss measures for transformations on the space of interval exchange maps, Ann. Math. 115 (1982) 201-242. 
[V2] W. Veech, The metric theory of interval exchange transformations I,II,III, Amer. J. Math. 106 (1984) 1331-1421.

Department of Mathematics, University of Groningen, P.O. Box 800, 9700 AV Groningen, the Netherlands

E-mail address: bruin@math.rug.nl

$U R L:$ http://www.math.rug.nl/ bruin/

Centre de Physique Théorique, Institut de Mathématiques de Luminy, Fédération De Recherche des Unités de Mathématiques de Marseille and Université de la Méditerranée, Luminy, Case 907, F-13288 Marseille Cedex 9, France

E-mail address: troubetz@iml.univ-mrs.fr

$U R L:$ http://iml.univ-mrs.fr/ troubetz/ 